\title{
Umami taste components and their sources in Asian foods
}

\begin{abstract}
Umami, the fifth basic taste, is the inimitable taste of Asian foods. Several traditional and locally prepared foods and condiments of Asia are rich in umami. In this part of world, umami is found in fermented animal-based products such as fermented and dried seafood, and plant-based products from beans and grains, dry and fresh mushrooms, and tea. In Southeast Asia, the most preferred seasonings containing umami are fish and seafood sauces, and also soybean sauces. In the East Asian region, soybean sauces are the main source of umami substance in the routine cooking. In Japan, the material used to obtain umami in dashi, the stock added to almost every Japanese soups and boiled dishes, is konbu or dried bonito. This review introduces foods and seasonings containing naturally high amount of umami substances of both animal and plant sources from different countries in Asia.
\end{abstract}

Keyword: 5'-nucleotides; Asian foods; Glutamate; Taste active compounds; Umami 